\title{
Capacidad cardiorrespiratoria en estudiantes de educación superior: análisis de redes sociales de amigos universitarios
}

\author{
Cardiorespiratory capacity in higher education students: analysis of social \\ networks of university friends
}

\section{Capacidade cardiorrespiratória em estudantes de educação superior: análise de redes sociais de amigos universitários}

\author{
Jason Cardona Gómez'; Juan Fernando Saldarriaga Franco²; Mario Andrés Quintero Velásquez; Carlos Mario Arango Paternina ${ }^{4}$ \\ 1 Magíster en Epidemiología. Secretaría de Educación de Envigado. Envigado, Colombia. Jason.cardona@udea.edu.co. ORcid: https:// \\ orcid.org/0000-0002-0067-5986 \\ 2 Magíster en Epidemiología. Universidad de Antioquia. Medellín, Colombia. jfernando.saldarriaga@udea.edu.co. ORCID: https://orcid. \\ org/0000-0002-4982-7047 \\ 3 Médico. Especialista en Medicina Aplicada a la Actividad Física y al Deporte. Indeportes. Medellín, Colombia. mquint@une.net.co. \\ ORCID: https://orcid.org/0000-0003-2967-6509 \\ 4 Doctor in Physical Education and Health of University of West Florida. Universidad de Antioquia. Medellín, Colombia. carlos.arangop@ \\ udea.edu.co. ORCID: https://orcid.org/0000-0002-5263-3139
}

Recibido: 22/05/2020. Aprobado: 09/06/2021. Publicado: 14/07/2021

Cardona-Gómez J, Saldarriaga-Franco JF, Quintero-Velásque MA, Arango-Paternina CM. Capacidad cardiorrespiratoria en estudiantes de educación superior: análisis de redes sociales de amigos universitarios. Rev. Fac. Nac. Salud Pública. 2021;39(3):e341657. DoI: https://doi.org/10.17533/udea.rfnsp.e341657

\section{Resumen}

Objetivo: Evaluar la asociación entre las redes sociales de amigos y la capacidad cardiorrespiratoria en estudiantes universitarios. Metodología: Estudio de corte, con muestreo aleatorio de cursos completos, por cada programa y semestre académico, con 475 participantes, realizado durante 2018. Se utilizaron mediciones de peso, talla, índice de masa corporal y encuesta para determinar el nivel de actividad física, las actividades sedentarias y las métricas de red social (como densidad de la red, centralidad de la red, popularidad social, miembro de grupos, proporción de amigos activos físicamente y proporción de amigos con alta capacidad cardiorrespiratoria).

Resultados: Las variables de la red social presentan un comportamiento diferente según sexo. En las mujeres, respecto a la red social, aumentar $10 \%$ la proporción de amigos activos físicamente incrementa $150 \%$ la posibilidad de tener capacidad cardiorrespiratoria alta (OR: 2,50 IC:1,01-6,21); incrementar las relaciones sociales (centralidad de la red) disminuye la oportunidad de tener capacidad cardiorrespiratoria alta en $65 \%$ (OR: 0,35, IC:0,19-0,65); tener alta popularidad social acrecienta en $120 \%$ la oportunidad de tener capacidad cardiorrespiratoria alta (OR: 2,20, IC:1,07-4,55). En los hombres, no se encontró asociación entre las variables de la red social con la capacidad cardiorrespiratoria. Conclusiones: La capacidad cardiorrespiratoria es influenciada por la red social de amigos universitarios y su comportamiento difiere según sexo. La red social en la que se desenvuelven los sujetos es un medio en el que se comparten y trasmiten comportamientos saludables y no saludables.

---------Palabras clave: Redes sociales, universitarios, factores de riesgo, estilo de vida, actividad física. 


\begin{abstract}
Objective: To evaluate the association between the social networks of friends and the cardiorespiratory capacity in university students. Methodology: A cut-off study was carried out in 2018; with random sampling of complete course groups for each program and academic semester with 475 participants. Weight, height, body mass index, and a survey were used to determine the level of physical activity, sedentary activities, and social network metrics (such as network density, network centrality, social popularity, group membership, rate of physically active friends, and rate of friends with high cardiorespiratory capacity). Results: The variables of the social network show a different behavior according to sex. Regarding the social network in women, an increase in the rate of physically active friends by $10 \%$ increases the possibility of having high cardiorespiratory capacity by 150
\end{abstract}

\% (OR: 2.50, CI: 1.01-6.21); increasing social relationships (network centrality) decreases the probability of having high cardiorespiratory capacity by $65 \%$ (OR: 0.35 , CI: $0.19-0.65$ ); having high social popularity increases the chance of having high cardiorespiratory capacity by $120 \%$ (OR: 2.20, CI: $1.07-$ $4.55)$. In men, no association was found between the variables of the social network and the cardiorespiratory capacity. Conclusions: Cardiorespiratory capacity is influenced by the social network of university friends, and its behavior differs according to sex. The social network in which the subjects operate is a medium where healthy and unhealthy behaviors are shared and transmitted.

--------Keywords: social networks, university students, risk factors, lifestyle, physical activity.

\section{Resumo}

Objetivo: Avaliar a associação entre as redes sociais de amigos e a capacidade cardiorrespiratória em universitários. Métodos: Estudo de corte, com amostragem aleatória de cursos completos, para cada programa e semestre letivo, com 475 participantes, realizado durante 2018. Peso, altura, índice de massa corporal e medidas de levantamento foram utilizados para determinar o nível de atividade física. atividades sedentárias e métricas de rede social (como densidade de rede, centralidade de rede, popularidade social, associação a grupos, proporção de amigos fisicamente ativos e proporção de amigos com alta capacidade cardiorrespiratória). Resultados: As variáveis da rede social apresentam um comportamento diferente de acordo com o sexo. Nas mulheres, em relação à rede social, aumentar a proporção de amigos fisicamente ativos em $10 \%$ aumenta em $150 \%$ a possibilidade de ter alta capacidade cardiorrespiratória (OR: 2,50 IC: 1,01-6,21); aumentar as relações sociais (centralidade da rede) diminui a oportunidade de ter alta capacidade cardiorrespiratória em $65 \%$ (OR: 0,35, IC: $0,19-0,65$ ); Ter alta popularidade social aumenta a oportunidade de ter alta capacidade cardiorrespiratória em $120 \%$ (or: 2,20 , IC: $1,07-4,55$ ). Nos homens, não foi encontrada associação entre as variáveis da rede social com a capacidade cardiorrespiratória. Conclusões: A capacidade cardiorrespiratória é influenciada pela rede social de amigos da universidade e seu comportamento difere conforme o sexo. A rede social em que os sujeitos atuam é um meio em que comportamentos saudáveis e não saudáveis são compartilhados e transmitidos.

-------Palavras-chave: Redes sociais, universidade, fatores de risco, estilo de vida, atividade física.

\section{Introducción}

La capacidad cardiorrespiratoria (CC) es entendida como la habilidad para captar, transportar y metabolizar oxigeno [1] y es reconocida como un importante marcador de salud y adaptación al medio [2]. Es un atributo biológico que integra numerosos sistemas: musculoesquelético, hematocirculatorio, endocrinometabólico, psiconeurológico y cardiorrespiratorio [3]. Según diversas investigaciones, aumentar la capacidad aeróbica $3,5 \mathrm{~mL} / \mathrm{kg} / \mathrm{min}$ en personas sanas, disminuye la mortalidad un $13 \%[2,4,5]$. En los últimos años, se ha detectado la reducción de la CC en adultos y jóvenes [6,7]. $\mathrm{Si}$ bien los determinantes de la $\mathrm{CC}$ incluyen aspectos genéticos [3], fisiológicos [8], comportamentales [9] y ambientales [10], este descenso se atribuye a la reducción de la actividad física y al incremento del tiempo sedentario de los sujetos [11]. Otros aspectos, como los factores sociales, han sido poco explorados, entre estos, las diferentes redes sociales que un sujeto establece durante sus etapas de la vida.

La red social es un medio en el que se pueden transmitir ideas, comportamientos (consumo de alcohol, cigarrillo, marihuana; ser físicamente activo en adolescentes [10], al igual que en los adultos se puede propagar la obesidad [12], etc.), las emociones, como la depresión, la felicidad y el sentido de cooperación [13].

Una de esas redes sociales es la red social de amigos, entendida como aquellas personas con quienes se 
tiene una cercanía en grado de amistad, se reúnen con frecuencia, a menudo juegan, comparten y tienen un interés mutuo en la misma actividad (tertulia, académica, creativa) [11].

Un ejemplo sobre la relación entre las redes sociales y los comportamientos, por ejemplo, sedentarios en adolescentes y jóvenes, se presenta en una investigación realizada en 6 escuelas de Canadá, en niños y adolescentes entre 11 y 15 años, en la que se encontró que aumentar $10 \%$ la proporción de tener amigos activos físicamente incrementa entre 11 y $14 \%$ la oportunidad de ser físicamente activo. También indicó que tener un mayor número de relaciones sociales (densidad de la red $>12 \%$ ) duplica la oportunidad de ser sedentario [10].

Así, de estas relaciones sociales se pueden destacar algunas métricas interesantes para indagar, por cuanto estas variables han mostrado tener relación con la actividad física: la popularidad social, el número de relaciones sociales o densidad de la red, los miembros de grupos que conforman la red social, la centralidad de la red social y los atributos de los amigos, y en particular, el porcentaje de amigos activos físicamente, entre otros. [10].

Y dado que, a su vez, estas variables de las redes sociales de amigos están asociadas con un factor protector de enfermedades crónicas como la actividad física, es posible pensar en incluir aquellas, en los estudios sobre estas redes, en relación con la propagación de los factores de riesgo para las enfermedades crónicas, como la obesidad, la diabetes y las enfermedades cardiovasculares, etc. $[10,12,13]$. Este es un aspecto clave a considerar, ya que al poderse demostrar la asociación entre la actividad física y las variables sociales, se podría esperar que estas últimas se relacionen con el cambio de comportamiento como, por ejemplo, la capacidad cardiorrespiratoria, al igual que se presenten el cambio de ideas o emociones.

Al respecto, una investigación prospectiva indagó sobre la obesidad en una red social con familiares y amigos, con más de 12000 personas desde el año 1971 al 2003 (32 años de seguimiento). Los resultados indicaron que tener un amigo con obesidad incrementa en 57 $\%$ la posibilidad de padecerla. Además, si son amigos mutuos, es decir, ambos consideran al otro como amigo en la red social, la posibilidad de ser obeso aumenta el $171 \%$. Si un hermano padece obesidad, el riesgo que otro hermano la presente crece el $40 \%$, y, si un cónyuge padece obesidad, el riesgo que su pareja la presente se acrecienta en $37 \%$ [12].

También se han documentado los lazos de amistad según sobrepeso. Una investigación realizada en 2014 sugiere que los jóvenes sin sobrepeso tienen $30 \%$ más posibilidades de elegir un amigo sin sobrepeso; sin embargo, los jóvenes con sobrepeso eran indiferentes a elegir amigos según su estado de peso [14].
De igual forma, en otro estudio se sostiene que las personas con sobrepeso $\mathrm{u}$ obesidad, que tienen relaciones de amistad con amigos activos físicamente, aumentan la motivación respecto a la práctica de actividad física [15].

De esta manera, el objetivo del estudio fue evaluar la asociación entre las redes sociales de amigos y la CC en estudiantes universitarios. Para ello, se toman en cuenta variables sociodemográficas, antropométricas y de las redes sociales de amigos como densidad de la red de amigos, proporción de amigos activos físicamente, popularidad, centralidad y miembro de grupo.

Los hallazgos generan asociaciones que serán de gran utilidad para plantear estrategias de intervención y seguimiento en salud pública, para combatir las enfermedades crónicas no transmisibles desde un enfoque social.

\section{Metodología}

Estudio de corte cuantitativo, transversal de asociación, donde se realiza una medición de una serie de variables en un solo momento y se establecen asociaciones entre estas [16].

\section{Población y muestreo}

La población estuvo conformada por estudiantes de pregrado de la Universidad CES, sede Medellín (segundo a octavo semestre), de carácter privada, pertenecientes a los programas de Medicina, Fisioterapia, Nutrición y Dietética, Biología y Química Farmacéutica.

Para la determinación del tamaño muestral, se empleó el software Epidat V.4.1.Este es un programa de libre distribución, dirigido a profesionales de la salud, con los siguientes criterios:

- Población: 3000 estudiantes.

- Proporción esperada de sobrepeso para población adulta: $35,3 \%$ [17].

- Nivel de confianza: $95 \%$.

- Error máximo permitido: $5 \%$.

- Tamaño de muestra: 407 estudiantes; finalmente, participaron en el estudio 475 estudiantes.

- Sobreestimación aproximada: $30 \%$ (anticipando pérdidas y no respuesta).

Para la obtención de la muestra se hizo selección aleatoria de salones completos (cursos) por cada programa y semestre académico. Se obtuvo el permiso institucional.

La recolección y el análisis de los datos se efectuó durante el 2018.

\section{Mediciones}

Se obtuvieron datos de variables sociodemográficas, antropométricas, $\mathrm{CC}$, comportamiento sedentario, nivel de actividad física y variables de la red social de los participantes, que se describen a continuación. 
- Variables sociodemográficas: edad, sexo, años de escolaridad, semestre cursado, estado civil, situación laboral y nivel socioeconómico. Se obtuvieron por encuesta.

El estrato socioeconómico se midió por el índice de bienes, el cual se elabora basado en la cantidad de bienes del hogar: casa propia, electricidad, baño interno, inodoro, conexión a internet, nevera, teléfono celular, teléfono fijo, lavadora, computador, tableta, televisión, aire acondicionado y carro [18].

De acuerdo con la cantidad de bienes, se calculan terciles para la clasificación y los análisis; de esta forma, se estableció el nivel socioeconómico alto, medio y bajo de los estudiantes [18].

- Variables antropométricas: el peso se midió con balanza Omron ${ }^{\circledR}$ (referencia: HBF-510LA); la estatura se determinó con cinta métrica inextensible, sin zapatos, soportando el peso en ambos pies y al final de la espiración; el índice de masa corporal (IMC) se calculó dividiendo el peso en kilogramos $(\mathrm{kg})$ por la estatura en metros al cuadrado $\left(\mathrm{m}^{2}\right)$. Se clasificaron las personas con peso normal: IMC $<25,0 \mathrm{~kg} / \mathrm{m}^{2}$, y sobrepeso y obesidad: $\mathrm{IMC} \geq 25,0 \mathrm{~kg} / \mathrm{m}^{2}$, según criterio de la Organización Mundial de la Salud (oms) [19].

La circunferencia de cintura se tomó según criterios de la oms [20]. Se utilizó una cinta métrica inextensible. Se clasificó la obesidad abdominal (OA) en hombres y mujeres, con valores de $91 \mathrm{~cm}$ y $89 \mathrm{~cm}$, respectivamente, según valores en población colombiana [21].

- Capacidad cardiorrespiratoria (variable dependiente): la CC se estimó según el modelo de predicción propuesto por Jurca et al. [22]. Para su determinación, se requiere: la edad en años, el IMC, la frecuencia cardíaca de reposo, el sexo y la clasificación del patrón de actividad característico, según autorreporte (cuestionario con 5 categorías/niveles de actividad física) [23]. Con estos datos, se obtiene la $\mathrm{CC}$, o el consumo máximo de oxígeno $\left(\mathrm{VO}_{2}\right.$ máx) en mililitros por kilogramo por minuto ( $\mathrm{mL} / \mathrm{kg} / \mathrm{min}$ ).

- Comportamiento sedentario: se utilizó el cuestionario Adolescent Sedentary Activity Questionnaire. Este ha sido utilizado para evaluar el comportamiento sedentario en población de estudiantes y categoriza las actividades sedentarias en 5 dominios: 1) pantalla pequeña, 2) educación, 3) viaje, 4) actividades culturales y $5)$ actividades sociales. Con esto se indica la frecuencia (días / sem) y la duración (horas / min / día).

Se clasificó como sedentaria, aquella persona que acumuló más de dos horas al día en actividades sedentarias [24,25].

- Nivel de actividad física: se utilizó el cuestionario Global Physical Activity Questionary para estimar el nivel de actividad física [26]. Cuenta con 5 preguntas, que indagan sobre la actividad realizada en tres dominios: 1) trabajo, 2) medio de transporte y
3) tiempo libre (cuestionario validado en estudiantes universitarios colombianos [27].

Se clasificaron como físicamente activas aquellas personas que realizaban mayor o igual que 150 minutos semanales de actividad física moderada o 75 minutos de actividad física intensa [28].

- Variables de la red social de amigos: se le pidió a cada individuo nominar o indicar a sus mejores amigos dentro de la universidad. La operacionalización de "mejor amigo" se definió como: aquellas personas con quienes se tiene una cercanía en grado de amistad en la universidad, se reúnen con frecuencia, a menudo juegan o comparten la misma actividad $\mathrm{y}$ tienen un interés compartido en alguna actividad (tertulia, académica, creativa).

Con esta información se extrajeron 6 métricas sobre la red social de amigos, teniendo en cuenta los amigos de la red establecida en la muestra para hacer un análisis egocéntrico (estructura de la red de cada individuo), así:

1. Número total de relaciones existentes sobre el total posible en la red (densidad). Esta se mide en términos porcentuales de 0 a 100 .

2. Proporción de nominaciones hechas y recibidas de personas que alcanzaron niveles recomendados de actividad física (proporción de amigos cercanos activos). Esta se mide en términos porcentuales de 0 a 100.

3. Proporción de nominaciones hechas y recibidas de personas con alta $\mathrm{CC}$ (proporción de amigos cercanos con alta $\mathrm{CC}$ ). Esta se mide en términos porcentuales de 0 a 100.

4. Cantidad de veces que una persona se encuentra en la ruta más corta entre dos personas (centralidad en la red). Se mide en números absolutos, según grupos.

5. Número total de nominaciones recibidas (popularidad). Se mide en números absolutos y corresponde a personas.

6. Conexiones con al menos otros dos amigos, y los tres se conectan a través de nominaciones de amistad (miembro de grupo). Se mide en términos absolutos [29].

\section{Análisis estadístico}

Se realizó la prueba de normalidad Shapiro-Wilk, para determinar la distribución de variables cuantitativas.

Las variables con distribución normal se presentan con medias y desviación estándar; las variables no normales se resumen con medianas y rangos intercuartílicos (RI).

La CC se dicotomizó en capacidad cardiorrespiratoria alta (CCA), así: hombres ( $>50,2 \mathrm{~mL} / \mathrm{kg} / \mathrm{min})$; mujeres $(>39,5 \mathrm{~mL} / \mathrm{kg} / \mathrm{min}$ ), según la mediana (prueba de normalidad realizada previamente).

Las variables cualitativas se resumieron con proporciones. Para las mujeres, la alta densidad de la red $(>41,1$ $\%$ ), la alta centralidad $(>1)$, la alta popularidad $(>3)$ y el miembro de grupo $(>4)$ fueron dicotomizados según la 
mediana. Para los hombres, las variables fueron dicotomizadas así: $>33,3,>2,>3$ y $>4$, respectivamente.

La proporción de amigos activos físicamente y la de amigos con CCA se miden en términos porcentuales y se aproximaron en los análisis multivariados en unidad de cambio de 10 puntos porcentuales (0\%-10\% - $20 \%$ $30 \%-40 \%$, etc.).

La CCA se analizó para cada sexo. Se hicieron pruebas chi-cuadrado $\left(\chi^{2}\right)$ de independencia según sobrepeso (sí-no), sedentarismo (sí-no), obesidad abdominal (síno), popularidad social (alta-normal), densidad de la red (alta-normal), centralidad de la red (alta-normal), miembro de grupo (alta-normal), nivel socioeconómico (bajomedio-alto) y programa académico.

Se aplicó la prueba U de Mann Whitney al comparar la variable dependiente con la proporción de amigos activos físicamente y la proporción de amigos con CCA.

Se realizaron modelos de regresión logística binaria multivariada, para hombres y mujeres, a razón de las diferencias según sexo, tanto para la variable dependiente como para las variables explicativas. En ambos modelos, el desenlace fue: CCA (sí-no).

Las variables candidatas a ingresar a los modelos fueron elegidas de acuerdo con el criterio de HosmerLemeshow (valor $p<0,25$ ). También las variables de la red social de amigos se ingresaron según literatura [10]. La variable sobrepeso se ingresó en el modelo para las mujeres, porque no se presentaron casos de obesidad abdominal en el grupo que tenía CCA. En el caso de los hombres, no se ingresó la variable sobrepeso, sino obesidad abdominal.

\section{Análisis de interacciones y confusión}

Se hizo análisis de interacción entre las variables de la red social: densidad de la red, miembro de grupo, centralidad de la red, popularidad de la red, y variables como edad, nivel socioeconómico, obesidad abdominal, sobrepeso y nivel socioeconómico, programa académico y semestre. No se encontraron interacciones (datos no mostrados). El modelo de regresión logística permitió obtener la razón de probabilidades (Odds Ratios, OR) ajustados, considerando múltiples covariables.

En todos los análisis, el nivel de significación estadística fue del $5 \%(p<0,05)$. Para el análisis de los datos se utilizó el software SPSS ${ }^{\circledR}$ Stadistics IBM ${ }^{\circledR}$ V.22 (Chicago) y Excel ${ }^{\circledR}$ 2013. Para el análisis de las variables sociales se utilizó el software UCINET ${ }^{\circledR}$ V.6 (Cambridge). Este software libre es especializado para realizar análisis de redes sociales; para utilizar este software, es necesario crear una matriz binaria en Excel $\AA$, para luego crear las métricas de las redes sociales [30].

\section{Criterios éticos}

El protocolo fue aprobado por el Comité de Ética del Instituto Universitario de Educación Física - Universi- dad de Antioquia (Acta 42 del 22 de marzo 2018). También fue aprobado por las coordinaciones académicas de los pregrados de la Universidad CES, sede Medellín.

La declaración de Helsinki celebrada en Fortaleza [31] y la Resolución 8430 de 1993 del Ministerio de la Protección Social de la Republica de Colombia sobre investigaciones con seres humanos en Colombia [32] fueron los parámetros que se tuvieron en cuenta para garantizar la protección de la intimidad.

Según la Resolución 8430, la investigación se clasifica como de riesgo mínimo [32].

\section{Resultados}

Se seleccionaron 563 estudiantes. De ellos, 88 fueron excluidos del estudio. Así, la muestra efectiva fue de 475 estudiantes (339 mujeres: 71,4\%), con una mediana de edad de 19 años (RI: 18-21) (véase Figura 1).

Las medianas del peso, talla e IMC fueron $59,3 \mathrm{k}$, $1,63 \mathrm{~m}$ y $22,3 \mathrm{~kg} / \mathrm{m}^{2}$, respetivamente. La mediana del tiempo destinado en actividades sedentarias a la semana fue de 1660 minutos por persona. En términos porcentuales, tres de cada cuatro $(75 \%)$ estudiantes destinaron más de 2 horas diarias a actividades sedentarias y el $49,5 \%$ presentó CCA.

El 20,8 \% de los estudiantes mostró sobrepeso según IMC, y el 5,3\%, obesidad abdominal.

En cuanto al nivel socioeconómico categorizado en bajo, medio y alto, las proporciones fueron: $36,6,22,5 \mathrm{y}$ $40,8 \%$, respectivamente.

Más del $50 \%$ de la muestra fueron nominados por otras personas 3 veces (popularidad). El $50 \%$ de los estudiantes del estudio integraron al menos 4 grupos de amigos diferentes (miembro de grupo), independiente del programa. La mediana de la centralidad de la red fue de 1 . La mediana de la proporción de amigos con CCA fue de $50 \%$, así como la mediana de la proporción de amigos activos físicamente fue de $80 \%$.

Dadas las diferencias entre hombres y mujeres en las variables antropométricas, la CC y la red social, los análisis bivariados y multivariados se estratificaron por sexo.

Respecto a las variables sociales, se encontró en las mujeres un aumento en el número de relaciones sociales en la red (densidad), comparado con los hombres, aunque estos presentaron mayor proporción de amigos activos físicamente.

No se hallaron diferencias por sexo en la centralidad de la red, el miembro de grupo, la proporción de amigos con CCA y la popularidad (véase Tabla 1).

En el análisis bivariado realizado en mujeres, hubo diferencias estadísticamente significativas entre la proporción de amigos con CCA y la posibilidad de tener CCA; asimismo, las personas con CCA presentaron más 


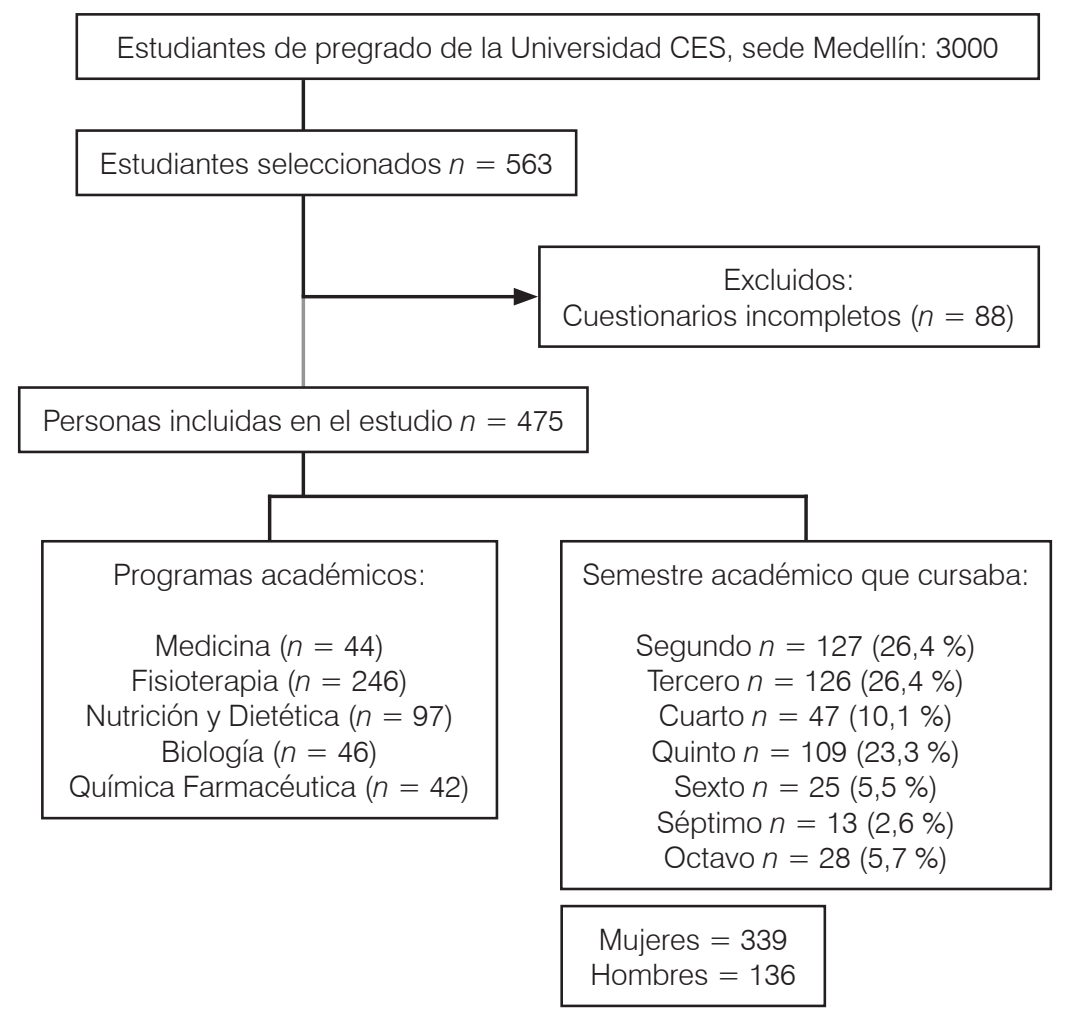

Figura 1. Flujograma selección de la muestra, estudiantes pregrado, Universidad cEs, sede Medellín

proporción de amigos activos físicamente que las personas con CC normal.

Se encontraron mayores proporciones de CCA en los niveles socioeconómicos medios y altos, comparado con el nivel bajo. Las personas con mayor centralidad en la red presentaron menores proporciones de CCA.

Ninguna persona con OA presentó CCA, mientras que, en aquellos sin OA, alrededor de la mitad tenían CCA.

En los programas de Nutrición y Química Farmacéutica se hallaron mayores proporciones de CCA.

Finalmente, aquellas personas con sobrepeso presentaron proporciones muy bajas de CCA, comparadas con aquellas sin esta condición (véase Tabla 2).

En cuanto al análisis realizado en hombres, de las personas con OA, cerca de la quinta parte presentó CCA, mientras que, en aquellos sin OA, más de la mitad presentó dicha condición favorable. Al comparar la proporción de CCA en personas con y sin sobrepeso, fue el doble en las personas sin sobrepeso (véase Tabla 2).

Las variables explicativas que integran el modelo logístico para las mujeres fueron estadísticamente significativas. Se halló que aumentar $10 \%$ la proporción de amigos activos físicamente aumenta $150 \%$ la oportunidad de tener CCA. También, encontrarse más de una vez en la ruta más corta entre dos personas (alta centralidad), disminuye $65 \%$ la posibilidad de tener CCA. Tener popularidad alta aumenta en $120 \%$ la oportunidad de tener CCA. Pertenecer al nivel socioeconómico medio y alto aumenta 503 y $300 \%$, respectivamente, la oportunidad de tener CCA, comparados con el nivel bajo. Los programas de Fisioterapia y Medicina fueron factores donde se disminuyó la posibilidad de tener CCA (véase Tabla 3).

Las variables explicativas que integran el modelo logístico para los hombres fueron estadísticamente significativas. Se encontró que padecer de obesidad disminuye el $80 \%$ la posibilidad de tener CCA. La proporción de amigos activos físicamente, la proporción de amigos con CCA, la centralidad de la red, la popularidad y el nivel socioeconómico no se asociaron con la variable dependiente (véase Tabla 3).

\section{Discusión}

El objetivo del estudio fue evaluar la asociación entre las redes sociales de amigos (densidad de la red de amigos, proporción de amigos activos físicamente, popularidad, centralidad y miembro de grupo) y la $\mathrm{CC}$ en estudiantes universitarios. La alta prevalencia de actividades sedentarias por más de 2 horas diarias es consistente con un estudio realizado en universitarios de Popayán [33]. Los resultados en mujeres sugieren, como factores que aumentan la CCA, la proporción de amigos activos físicamente, la popularidad social y el nivel socioeconómico medio y alto; a diferencia de la centralidad de la red, el sobrepeso y los programas de Fisioterapia y Medicina, que indicaron ser factores asociados a la disminución de la CCA. 
Tabla 1. Medidas de resumen para características sociodemográficas y redes sociales por sexo, para estudiantes pregrado

\begin{tabular}{|c|c|c|c|c|c|c|c|}
\hline \multirow{2}{*}{ Variable } & \multicolumn{3}{|c|}{$\begin{array}{c}\text { Mujeres } \\
(n=339)\end{array}$} & \multicolumn{3}{|c|}{$\begin{array}{l}\text { Hombres } \\
(n=136)\end{array}$} & \multirow{2}{*}{$\begin{array}{c}\text { Estadístico } \\
\text { (p) }\end{array}$} \\
\hline & Mediana & Q1 & Q3 & Mediana & Q1 & Q3 & \\
\hline Edad (años) & 19 & 18 & 21 & 19 & 18 & 20 & $0,267^{*}$ \\
\hline Peso (kg) & 55,9 & 51,7 & 61,5 & 69,6 & 63,2 & 78,8 & $<0,001^{*}$ \\
\hline Talla (m) & 1,60 & 1,55 & 1,64 & 1,72 & 1,68 & 1,78 & $<0,001^{*}$ \\
\hline $\operatorname{IMC}\left(\mathrm{kg} / \mathrm{m}^{2}\right)$ & 21,7 & 20,1 & 23,8 & 23,6 & 21,4 & 26,3 & $<0,001^{*}$ \\
\hline Actividades sedentarias ( $\mathrm{min}$ / sem) & 1560 & 780 & 2690 & 1930 & 1087 & 3234 & $0,009^{*}$ \\
\hline Cintura $(\mathrm{cm})$ & 71 & 67 & 76 & 80,4 & 74,2 & 85,9 & $<0,001^{*}$ \\
\hline $\mathrm{VO}_{2} \operatorname{máx}(\mathrm{mL} / \mathrm{kg} / \mathrm{min})$ & $39,5^{\dagger}$ & \multicolumn{2}{|c|}{$5,1^{\ddagger}$} & $50,2^{+}$ & \multicolumn{2}{|c|}{$4,9^{\ddagger}$} & $<0,001^{\S}$ \\
\hline Densidad de la red (\%) & 41,1 & 15,0 & 75,0 & 33,3 & 8,3 & 50,0 & $0,021^{*}$ \\
\hline Miembro de grupo & 4 & 1 & 11 & 4 & 1 & 9 & $0,851^{*}$ \\
\hline Centralidad de red & 1 & 0 & 4,2 & 2 & 0 & 6,0 & $0,09^{*}$ \\
\hline Popularidad en la red & 3 & 2 & 4 & 3 & 1,2 & 4,0 & $0,90^{*}$ \\
\hline Proporción de amigos con CCA (\%) & 50 & 30 & 70 & 50 & 30 & 78 & $0,15^{*}$ \\
\hline Proporción amigos activos (\%) & 80 & 50 & 100 & 95 & 70 & 100 & $0,006^{*}$ \\
\hline
\end{tabular}

CCA: Capacidad cardiorrespiratoria alta. IMC: Índice de masa corporal; Q1: Cuartil 1, Q3: Cuartil 3; $\mathrm{VO}_{2}$ máx Consumo de oxígeno máximo.

* Estadístico U de Mann Whitney.

$\dagger$ Media.

$\ddagger$ Desviación estándar.

$\S$ Diferencia de medias en muestras independientes.

Tabla 2. Análisis bivariado según capacidad cardiorrespiratoria alta y covariables, estudiantes pregrado

\begin{tabular}{|c|c|c|c|c|c|c|c|}
\hline \multirow[t]{2}{*}{ Variables } & & \multicolumn{2}{|l|}{$\begin{array}{c}\text { cca } \\
\text { Mujeres }\end{array}$} & \multirow[t]{2}{*}{ Estadístico $\chi^{2}$} & \multicolumn{2}{|l|}{$\begin{array}{c}\text { cca } \\
\text { Hombres }\end{array}$} & \multirow[t]{2}{*}{ Estadístico $\chi^{2}$} \\
\hline & & Proporción & $n$ & & Proporción & $n$ & \\
\hline \multirow{2}{*}{ Miembro de grupo } & Alto & 47,9 & 79 & \multirow{2}{*}{$0,08$ (1 gl. $p=0,78)$} & 51,6 & 33 & \multirow{2}{*}{$<0,001$ (1 gl. $p=0,98$} \\
\hline & Normal & 49,4 & 86 & & 51,4 & 37 & \\
\hline \multirow{2}{*}{ Centralidad de la red } & Alta & 40,8 & 64 & \multirow{2}{*}{ 7,32 (1 gl. $p=0,007)$} & 51,7 & 31 & \multirow{2}{*}{$0,002$ (1 gl. $p=0,97)$} \\
\hline & Normal & 55,5 & 101 & & 51,3 & 39 & \\
\hline \multirow{2}{*}{ Popularidad social } & Alta & 50,8 & 62 & \multirow{2}{*}{$0,35$ (1 gl. $p=0,56)$} & 54 & 27 & \multirow{2}{*}{$0,23$ (1 gl. $p=0,20)$} \\
\hline & Normal & 47,5 & 103 & & 50 & 43 & \\
\hline \multirow{2}{*}{ Densidad de la red } & Alta & 51,2 & 87 & \multirow{2}{*}{$0,86$ (1 gl. $p=0,36)$} & 54,7 & 41 & \multirow{2}{*}{$0,68$ (1 gl. $p=0,41)$} \\
\hline & Normal & 46,2 & 78 & & 47,5 & 29 & \\
\hline \multirow{2}{*}{ Proporción amigos con CCA } & CCA Sí & 50 & 174 & \multirow{2}{*}{$0,003^{*}$} & 55 & 70 & \multirow{2}{*}{$0,066^{\star}$} \\
\hline & CCA No & 40 & 165 & & 50 & 66 & \\
\hline \multirow{2}{*}{$\begin{array}{l}\text { Proporción amigos activos } \\
\text { físicamente }\end{array}$} & CCA Sí & 80 & 174 & \multirow{2}{*}{$0,004^{*}$} & 100 & 70 & \multirow{2}{*}{$0,144^{*}$} \\
\hline & CCA No & 70 & 165 & & 80 & 66 & \\
\hline \multirow{3}{*}{ Nivel socioeconómico } & Bajo & 34,4 & 42 & \multirow{3}{*}{$6,8(1 \mathrm{gl} . p=0,009)^{\dagger}$} & 53,8 & 28 & \multirow{3}{*}{$3,5$ (2 gl. $p=0,17)$} \\
\hline & Medio & 66,2 & 51 & & 63,3 & 19 & \\
\hline & Alto & 51,4 & 72 & & 42,6 & 23 & \\
\hline
\end{tabular}




\begin{tabular}{|c|c|c|c|c|c|c|c|}
\hline \multirow[t]{2}{*}{ Variables } & & \multicolumn{2}{|l|}{$\begin{array}{c}\text { cca } \\
\text { Mujeres }\end{array}$} & \multirow[t]{2}{*}{ Estadístico $\chi^{2}$} & \multicolumn{2}{|l|}{$\begin{array}{c}\text { cca } \\
\text { Hombres }\end{array}$} & \multirow[t]{2}{*}{ Estadístico $\chi^{2}$} \\
\hline & & Proporción & $n$ & & Proporción & $n$ & \\
\hline \multirow{5}{*}{ Programa } & FISIO & 42,8 & 71 & \multirow{5}{*}{$15,1$ (4 gl. $p=0,004)$} & 52,5 & 42 & \multirow{5}{*}{ 1,4 (4 gl. $p=0,82)$} \\
\hline & QF & 63,3 & 19 & & 41,7 & 5 & \\
\hline & MED & 34,4 & 11 & & 41,7 & 5 & \\
\hline & $\mathrm{BIOL}$ & 40,7 & 11 & & 52,6 & 10 & \\
\hline & NUTR & 63,1 & 53 & & 61,5 & 8 & \\
\hline \multirow{2}{*}{ Obesidad abdominal } & Sí & 0 & 0 & \multirow{2}{*}{$p=0,004^{\ddagger}$} & 18,8 & 3 & \multirow{2}{*}{$p=0,007^{\ddagger}$} \\
\hline & No & 49,8 & 163 & & 55,8 & 67 & \\
\hline \multirow{3}{*}{ IMC } & $\begin{array}{l}\text { Peso } \\
\text { normal }\end{array}$ & 54,2 & 154 & \multirow{3}{*}{ 21,6 (1 gl. $p<0,001)$} & 62 & 57 & \multirow{3}{*}{$12,5$ (1 gl. $p<0,001)$} \\
\hline & Sobrepeso & 20 & 11 & & 29,5 & 13 & \\
\hline & CCA no & 70 & 165 & & 80 & 66 & \\
\hline
\end{tabular}

CCA: Capacidad cardiorrespiratoria alta; gl.: grados de libertad; FISIO: Fisioterapia; QF: Química Farmacéutica; MED: Medicina; BIOL: Biología; NUTR: Nutrición y Dietética; IMC: Índice de masa corporal.

* Estadístico U de Mann Whitney.

$\dagger$ Prueba exacta de Fisher.

$\$$ Asociación lineal por lineal. Edad y capacidad cardiorrespiratoria baja: $p=0,798$. U de Mann Whitney.

Tabla 3. Modelos de regresión logística binaria según sexo, estudiantes pregrado

\begin{tabular}{|c|c|c|c|c|c|c|c|c|}
\hline & \multirow{2}{*}{\multicolumn{2}{|c|}{ Variables según sexo }} & \multirow{2}{*}{$\begin{array}{c}\text { OR } \\
\text { crudo }\end{array}$} & \multicolumn{2}{|c|}{ Ic95 \% para oR } & \multirow{2}{*}{$\begin{array}{c}\text { OR } \\
\text { Ajustado }\end{array}$} & \multicolumn{2}{|c|}{ Ic95 \% para or } \\
\hline & & & & Inferior & Superior & & Inferior & Superior \\
\hline \multirow{19}{*}{ 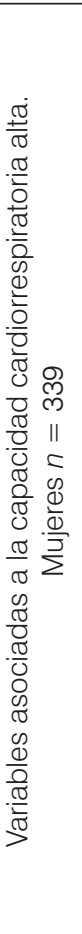 } & \multicolumn{2}{|c|}{ Amigos activos físicamente } & 2,57 & 1,29 & 5,11 & $2,50^{\star}$ & 1,01 & 6,21 \\
\hline & \multicolumn{2}{|c|}{ Amigos con capacidad cardiorrespiratoria alta } & 2,72 & 1,34 & 5,54 & 1,35 & 0,52 & 3,50 \\
\hline & \multicolumn{2}{|c|}{ Densidad de la red (> 41,1\%) } & 1,22 & 0,80 & 1,87 & 0,78 & 0,44 & 1,42 \\
\hline & \multicolumn{2}{|c|}{ Miembro de grupo en la red $(>4)$} & 0,94 & 0,61 & 1,43 & 0,83 & 0,41 & 1,68 \\
\hline & \multicolumn{2}{|c|}{ Centralidad de la red $(>1)$} & 0,55 & 0,36 & 0,85 & $0,35^{\star}$ & 0,19 & 0,65 \\
\hline & \multicolumn{2}{|l|}{ Popularidad social (> 3) } & 1,14 & 0,73 & 1,78 & $2,20 *$ & 1,07 & 4,55 \\
\hline & \multicolumn{2}{|l|}{ Sobrepeso IMC } & 0,21 & 0,11 & 0,43 & $0,16^{*}$ & 0,07 & 0,35 \\
\hline & \multirow{5}{*}{ Programa académico } & Nutrición & \multicolumn{3}{|c|}{1} & \multicolumn{3}{|c|}{1} \\
\hline & & Fisioterapia & 0,44 & 0,26 & 0,75 & $0,37^{\star}$ & 0,20 & 0,72 \\
\hline & & Química Farmacéutica & 1,01 & 0,43 & 2,40 & 1,70 & 0,65 & 4,47 \\
\hline & & Medicina & 0,31 & 0,13 & 0,72 & 0,21 & 0,08 & 0,57 \\
\hline & & Biología & 0,40 & 0,17 & 0,98 & 0,41 & 0,14 & 1,16 \\
\hline & \multirow{3}{*}{ Nivel socioeconómico } & Bajo & \multicolumn{3}{|c|}{1} & \multicolumn{3}{|c|}{1} \\
\hline & & Medio & 3,74 & 2,05 & 6,82 & $6,03^{*}$ & 2,94 & 12,31 \\
\hline & & Alto & 2,01 & 1,22 & 3,32 & $4,00^{*}$ & 2,14 & 7,49 \\
\hline & \multicolumn{8}{|c|}{ Estadístico Hosmer-Lemeshow: 0,667 } \\
\hline & \multicolumn{8}{|c|}{ Prueba de Ómnibus: $\chi^{2}:$ 87,86 (13 gl) $p<0,001$} \\
\hline & \multicolumn{8}{|l|}{$R^{2}$ de Nagelkerke: 0,305 } \\
\hline & \multicolumn{8}{|l|}{$\mathrm{R}^{2}$ de Cox y Snell: 0,228 } \\
\hline
\end{tabular}




\begin{tabular}{|c|c|c|c|c|c|c|c|c|}
\hline & \multirow{2}{*}{\multicolumn{2}{|c|}{ Variables según sexo }} & \multirow{2}{*}{$\begin{array}{c}\text { OR } \\
\text { crudo }\end{array}$} & \multicolumn{2}{|c|}{ IC95 \% para OR } & \multirow{2}{*}{$\begin{array}{c}\text { OR } \\
\text { Ajustado }\end{array}$} & \multicolumn{2}{|c|}{ IC95 \% para oR } \\
\hline & & & & Inferior & Superior & & Inferior & Superior \\
\hline \multirow{11}{*}{ 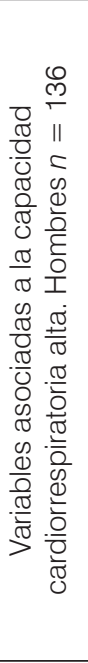 } & \multicolumn{2}{|c|}{ Amigos activos físicamente } & 1,95 & 0,61 & 6,24 & 1,22 & 0,30 & 5,03 \\
\hline & \multicolumn{2}{|c|}{ Amigos con capacidad cardiorrespiratoria alta } & 2,51 & 0,85 & 7,42 & 2,27 & 0,64 & 8,07 \\
\hline & \multicolumn{2}{|c|}{ Centralidad de la red $(>2)$} & 1,01 & 0,52 & 1,99 & 0,97 & 0,36 & 2,58 \\
\hline & \multicolumn{2}{|c|}{ Popularidad de la red $(>3)$} & 1,17 & 0,58 & 2,36 & 1,18 & 0,42 & 3,31 \\
\hline & \multicolumn{2}{|c|}{ Densidad de la red (> 33 \%) } & 1,33 & 0,68 & 2,62 & 1,37 & 0,62 & 3,04 \\
\hline & \multicolumn{2}{|c|}{ Miembro de grupo en la red $(>4)$} & 1,01 & 5,13 & 1,98 & 0,83 & 0,33 & 2,09 \\
\hline & \multicolumn{2}{|l|}{ Obesidad abdominal } & 0,18 & 0,05 & 0,67 & $0,20 *$ & 0,05 & 0,78 \\
\hline & \multirow{3}{*}{ Nivel socioeconómico } & Bajo & \multicolumn{3}{|c|}{1} & \multicolumn{3}{|c|}{1} \\
\hline & & Medio & 1,48 & 0,59 & 3,72 & 1,29 & 0,49 & 3,43 \\
\hline & & Alto & 0,64 & 0,29 & 1,37 & 0,63 & 0,28 & 1,41 \\
\hline & \multicolumn{2}{|c|}{$\begin{array}{l}\text { Estadístico Hosmer-Lemeshow: 0,092 } \\
\text { Prueba de Ómnibus: } \chi^{2}: 14,26(9 \mathrm{gl}) p=0,113 \\
\mathrm{R}^{2} \text { de Nagelkerke: } 0,133 \\
\mathrm{R}^{2} \text { de Cox y Snell: } 0,10\end{array}$} & & & & & & \\
\hline
\end{tabular}

$\mathrm{IMC}=$ Índice de masa corporal; OR: Odds Ratio. IC = Intervalo de confianza. Los oR que se resaltan en gris refieren a valores que son estadísticamente significativos significativos

* Los OR son estadísticamente significativos

El porcentaje de amigos activos físicamente hace que los amigos cercanos con este atributo generen una red social donde se propicia la adopción de estilos de vida saludables y es ahí cuando este tipo de amigos influyen en la modificación del comportamiento. Una investigación de tipo transversal concluyó que aumentar en $10 \%$ la proporción de amigos activos físicamente incrementa la posibilidad de ser activo físicamente [10]; y un estudio prospectivo (32 años de seguimiento) determinó la causalidad de la red social de las personas, incluida la familia, en la propagación de la obesidad [12]. A la fecha, se sugiere que la red social y familiar posibilitan la propagación de comportamientos, emociones o ideas [13], situación evidenciada en este estudio.

En las mujeres, aumentar la proporción de amigos activos físicamente incrementa la oportunidad de tener CCA, resultados similares a los encontrados en otra investigación, donde se indica que aumentar en $10 \%$ la proporción de amigos activos físicamente incrementa entre 11 y $14 \%$ la oportunidad de ser físicamente activo [10]. Aunque el estudio citado no cuantificó la CC, sí encontró relación entre el nivel de actividad física individual y la proporción de amigos activos físicamente. Con precaución se podría considerar que aumentar la proporción de amigos activos físicamente conduciría a un incremento de la CC.

Igualmente, se encontró que, en las mujeres, ser popular socialmente (ser nominado más de tres veces por alguien) se asoció con un aumento de la CCA. Esta situación se puede explicar por el hecho de que las mujeres que participaron en este estudio, fueron personas activas físicamente $(70,2 \%)$ y al establecer más relaciones sociales, la red social se conformaba principalmente por personas activas físicamente, situación que puede resultar provechoso para asumir estilos de vida activos y mejorar la CC. Además, según Christakis y Fowler [13], la popularidad social depende de tener un atributo que hace que alguien se destaque socialmente; en este caso, se puede pensar que si alguien tiene CCA, podría ser más popular.

Algunos estudios han encontrado la asociación entre las variables de las redes sociales y el nivel de actividad física para ambos sexos $[10,34]$, situación que en nuestra investigación no se evidenció en los hombres. Al respecto, debe considerarse el menor tamaño muestral en este grupo y sus implicaciones, en términos de la reducción de la potencia estadística y el aumento de la imprecisión en las estimaciones de las asociaciones.

También se halló que, en las mujeres, pertenecer a los niveles socioeconómicos medio y alto incrementa la oportunidad de tener CCA, en comparación con el nivel bajo. Según una investigación realizada en Medellín, las personas con mayores ingresos económicos tienen mayor oferta en cuanto a tipos de actividad física, como los que se ofrecen en los gimnasios, mejor infraestructura y mayor variedad en cuanto a escenarios deportivos, mejorando su acceso y frecuencia de realización [35]. Esta situación implica aumentos de la CC de estos grupos poblacionales.

Como factores de riesgo, se identificó que tener más conectividad en la red social (centralidad) tiende a disminuir la posibilidad de tener CCA. Esto se puede 
explicar por el hecho de que las personas están más conectadas en la red y se tiende a destinar más tiempo en las relaciones sociales y no a la actividad física. Estos resultados son similares a un estudio realizado en Canadá en adolescentes, donde se encontró que, en los hombres, tener alta densidad de la red social o alto número de relaciones sociales aumenta cerca de $200 \%$ el riesgo de ser sedentario [10].

El sobrepeso también se asoció con una disminución en la CCA. Este hallazgo es similar al encontrado por Rauner, Mess y Woll en una revisión sistemática en adolescentes de 11 a 19 años, donde se identificó una relación inversa entre el sobrepeso y la CC [36]. En los hombres, la obesidad se asoció con una menor $\mathrm{CC}$, hallazgo que concuerda con un estudio efectuado en adolescentes, donde los sujetos con CCA presentaron valores más bajos en los pliegues del tronco, comparados con aquellos con CC baja [37].

En las mujeres, pertenecer a los pregrados de $\mathrm{Me}$ dicina y Fisioterapia disminuyó la posibilidad de tener CCA, comparado con el pregrado de Nutrición. Este hallazgo se podría explicar, en parte, por las características curriculares de los programas, donde por los contenidos que son vistos en los cursos, puede hacer que los estudiantes prioricen la parte académica. Esto puede hacer que los estudiantes no participen en la oferta de actividades o programas de estilo de vida saludable en la Universidad en la que se aplicó el estudio. A la par, otras investigaciones han referido altos niveles de sedentarismo con valores del $77 \%$ en estudiantes de Medicina [38], y $97 \%$ en estudiantes de Fisioterapia [33]. En estas condiciones, es esperable la disminución de la $\mathrm{CC}$ y el incremento prematuro del riesgo de enfermedades crónicas, situación que exige atención.

Entre las limitaciones del estudio se encuentran el autorreporte como medio de obtención de algunos datos. Esto puede generar sesgos de información (memoria). También, la determinación de la $\mathrm{CC}$ fue indirecta, ya que se estimó mediante ecuación de predicción, sin la realización de una prueba de laboratorio (ergoespirometría). Asimismo, el nivel de actividad física y los comportamientos sedentarios se obtuvieron por medio de cuestionarios, lo que puede generar imprecisiones en las mediciones. Igualmente, la naturaleza transversal del estudio hace que no se pueda determinar la causalidad entre las variables de las redes sociales y la CCA. Del mismo modo, solo incluir amigos universitarios excluye del estudio personas como familiares $u$ otro tipo de amigos, que también pueden influenciar los comportamientos, situación evidenciada en otros estudios [12]. Incluso, el no incluir variables musculoesqueléticas, hematocirculatorias, endocrinometabólicas y psiconeurológicas, que son potenciales confusoras (ya que pueden modificar la capacidad cardiorrespiratoria), puede limi- tar los resultados del estudio. Finalmente, la baja proporción de hombres en la muestra es un factor que limita la exploración de las asociaciones en el modelo logístico. Futuras investigaciones deberán dilucidar la influencia potencial de las redes sociales en la CCA en hombres.

Entre las fortalezas del estudio se encuentran la alta tasa de respuesta de la red social (aproximadamente, el $65 \%$ ). Esto quiere decir que de las personas que nominaron y fueron nominadas en la red social, se obtuvieron datos del $65 \%$ en el total de la muestra, esto comparado con el cerca de $20 \%$ reportado en un estudio previo [10].

Otra fortaleza es la realización de las mediciones con estricto apego a un protocolo estandarizado por los investigadores.

Nuestro estudio señala la importancia de incorporar el análisis de redes para comprender el comportamiento de factores de riesgo comportamentales y del estilo de vida en jóvenes, esto mediante un estudio de intervención. Esto permitiría tener más herramientas para combatir las enfermedades crónicas, que hasta el momento aumentan cada vez más sus prevalencias.

La red social en la que se desenvuelven los sujetos es un medio en el que se comparten y trasmiten comportamientos saludables y no saludables, situación que señala la importancia de la utilización de las tecnologías de la información y la comunicación para la definición de enfoques renovados de promoción de la salud en los jóvenes, pasando de un enfoque intrapersonal al interpersonal.

\section{Conclusiones}

La CC es influenciada por la red social de amigos y su comportamiento difiere en hombres y mujeres. El presente estudio identificó asociaciones estadísticamente significativas en el caso de las mujeres.

Tener amigos activos físicamente incrementa la CCA. Asimismo, tener amigos con CCA acrecienta la posibilidad de que una persona aumente su CC.

Tener alta popularidad social incrementa la CCA.

El sobrepeso también disminuye la posibilidad de tener CCA, debido al aumento de grasa corporal.

Los resultados señalan la importancia del análisis de redes e invita a realizar estudios de intervención para el diagnóstico, el seguimiento y la intervención temprana de los factores de riesgo, con miras a la prevención de las enfermedades crónicas degenerativas. Los amigos pueden influir en comportamientos tanto saludables como no saludables; a sabiendas de ello, se pueden diseñar estrategias para maximizar los efectos saludables y minimizar los factores de riesgo para la salud.

Pertenecer a los niveles socioeconómicos medio y alto aumenta la CCA. Esto podría deberse al poder adquisitivo que se puede tener en esta condición, lo que puede posibilitar a estas personas acceder a ciertas actividades 
físicas a las que quizás no tengan acceso las personas con nivel socioeconómico bajo. Al respecto, este estudio solo puede inferir dicha asociación, por lo que se recomienda realizar futuras investigaciones sobre el tema.

Paradójicamente, los pregrados en ciencias de la salud son factores que disminuyen la CCA. Estos han reportado mayores factores de riesgo para enfermedades no transmisibles, como sobrepeso, inactividad física, entre otros, comparados con otros programas de pregrado. Se recomiendan futuras investigaciones para ahondar el tema.

Se recomienda hacer futuras intervenciones de la red social, con el fin de establecer la causalidad entre la red social de amigos y la CC. También se recomienda llevar a cabo investigaciones donde se indague qué tipos de actividad física realizan los amigos, o en qué contextos (puede ser que entre amigos se reúnan para hacer actividades en el gimnasio o al aire libre, o solo de tipo aeróbico). Esto ayudaría a tener más herramientas a la hora de diseñar programas de promoción de la salud y prevención de la enfermedad. También se recomienda estudiar otros tipos de relaciones sociales con amigos que puedan perjudicar las actividades físicas, como factor que permita emitir propuestas para la prevención de enfermedades.

\section{Conflicto de intereses}

Los autores de esta investigación declaran no tener conflicto de intereses.

\section{Fuentes de financiación}

Esta investigación contó con financiación del Comité para el Desarrollo de la Investigación, de la Universidad de Antioquia.

\section{Declaración de responsabilidad}

Los puntos de vista expresados en este artículo son responsabilidad de los autores.

\section{Contribución de los autores}

Este artículo presentó la contribución de los cuatro autores en cuanto a participación en el análisis e interpretación de los datos, también en el diseño de este. Todos participaron en la aprobación de la versión final de este artículo y todos están en capacidad de responder por todos los aspectos de este artículo, cumpliendo de esta manera con los cuatro criterios del Comité Internacional de Editores de Revistas Médicas (International Committee of Medical Journal Editors, ICMJE).

\section{Referencias}

1. Aspenes ST, Nilsen TI, Skaug EA, et al. Peak oxygen uptake and cardiovascular risk factors in 4631 healthy women and men. Med Sci Sports Exerc. 2011;43(8):1465-73. Dor: https://doi. org/10.1249/MSS.0b013e31820ca81c

2. Ross R, Blair SN, Arena R, et al. Importance of assessing cardiorespiratory fitness in clinical practice: A case for fitness as a clinical vital sign: A scientific statement from the American Heart Association. Circulation. 2016;134(24):e653-e99. DoI: https://doi. org/10.1161/CIR.0000000000000461

3. Hernández-Mosqueira C, Fernandes Da Silva S, Fernandes Filho J. Tablas de referencia de condición física en niñas de 10 a 14 años de Chillán, Chile. Rev Salud Pública. 2015;17(5):667-76. DOI: https://doi.org/10.15446/rsap.v17n5.41674

4. Nes BM, Vatten LJ, Nauman J. A simple nonexercise model of cardiorespiratory fitness predicts long-term mortality. Med Sci Sports Exerc. 2014;46(6):1159-65. DoI: https://doi.org/10.1249/ MSS.0000000000000219

5. Kokkinos PF, Faselis C, Myers J, et al. Interactive effects of fitness and statin treatment on mortality risk in veterans with dyslipidaemia: A cohort study. Lancet. 2013;381(9864):394-9. DoI: https://doi.org/10.1016/S0140-6736(12)61426-3

6. Venckunas T, Emeljanovas A, et al. Secular trends in physical fitness and body size in Lithuanian children and adolescents between 1992 and 2012. J Epidemiol Community Health. 2017;71(2):1817. Dor: https://doi.org/10.1136/jech-2016-207307

7. Costa AM, Costa MJ, Reis AA, et al. Secular trends in anthropometrics and physical fitness of young Portuguese school-aged children. Acta Med Port. 2017;30(2):108-14. DoI: https://doi. org/10.20344/amp.7712

8. Garzón M. La condición física es un componente importante de la salud para los adultos de hoy y del mañana. Selección [internet]. 2007 [citado 2019 may. 4]; 17(1):2-8. Disponible en: https://www. cafyd.com/selec0701/Selultimo.pdf

9. Martínez-Vizcaíno V, Sánchez-López M. Relación entre actividad física y condición física en niños y adolescentes. Rev Esp Cardiol. 2008;61(2):108-11. DoI: https://doi.org/10.1157/13116196

10. Sawka KJ, McCormack GR, et al. Associations between aspects of friendship networks and dietary behavior in youth: Findings from a systematized review. Eati Behav. 2015; 18:7-15. Dor: https://doi.org/10.1016/j.eatbeh.2015.03.002

11. Katzmarzyk PT. Physical activity, sedentary behavior, and health: Paradigm paralysis or paradigm shift? Diabetes. 2010;59(11):2717-25. DoI: https://doi.org/10.2337/db10-0822

12. Christakis NA, Fowler JH. The spread of obesity in a large social network over 32 years. N Engl J Med. 2007;357(4):370-9. Dor: https://doi.org/10.1056/NEJMsa066082

13. Christakis NA, Fowler JH. Social contagion theory: Examining dynamic social networks and human behavior. Stat Med. 2013;32(4):556-77. DoI: https://doi.org/10.1002/sim.5408

14. Schaefer DR, Simpkins SD. Using social network analysis to clarify the role of obesity in selection of adolescent friends. Am J Public Health. 2014;104(7):1223-9. DoI: https://doi.org/10.2105/ AJPH.2013.301768

15. Salvy SJ, Bowker JC, Germeroth L, Barkley J. Influence of peers and friends on overweight/obese youths' physical activity. Exerc Sport Sci Rev. 2012;40(3):127-32. DoI: https://doi.org/10.1097/ JES.0b013e31825af07b 
16. Grimes DA, Schulz KF. Descriptive studies: What they can and cannot do. Lancet. 2002; 359(9301):145-9. DoI: https://doi. org/10.1016/S0140-6736(02)07373-7

17. Fonseca Centeno Z, Heredia Vargas AP, Ocampo Tellez PR, et al ICBF. Encuesta nacional de la situación nutricional en Colombia 2010 ENSIN. Resumen Ejecutivo ENSIN [internet]; 2010 [citado 2016 dic. 20]. Disponible en: https:/www.icbf.gov.co/sites/default/files/resumenfi.pdf

18. Carrillo-Larco RM, Bernabé-Ortiz A, Pillay TD, et al. Obesity risk in rural, urban and rural-to-urban migrants: Prospective results of the PERU MIGRANT study. Int J Obes. 2016;40(1):181-5. DOI: https://doi.org/10.1038/ijo.2015.140

19. Organización Mundial de la Salud. Obesidad y sobrepeso [internet]; 2016 [citado 2017 ago. 17]. Disponible en: http://www.who. int/mediacentre/factsheets/fs $311 /$ es/

20. Organización Mundial de la Salud. Enfermedades no transmisibles y sus factores de riesgo. Ginebra [internet]; 2013 [citado 2018 dic. 25]. Disponible en: https://www.who.int/ncds/es/

21. Buendía R, Zambrano M, Díaz Á, et al. Puntos de corte de perímetro de cintura para el diagnóstico de obesidad abdominal en población colombiana usando bioimpedanciometría como estándar de referencia. Rev Colomb Cardiol. 2016;23(1):19-25. DoI: https:// doi.org/10.1016/j.rccar.2015.07.011

22. Jurca R, Jackson AS, LaMonte MJ, et al. Assessing cardiorespiratory fitness without performing exercise testing. Am J Prev Med. 2005;29(3):185-93. DoI: https://doi.org/10.1016/j.amepre.2005.06.004

23. Blair SN, Kohl HW, et al. Physical fitness and all-cause mortality: A prospective study of healthy men and women. JAMA [internet] 1989 [citado 2019 dic. 20]; 262(17):2395-401. Disponible en: https://pubmed.ncbi.nlm.nih.gov/2795824/

24. Hardy L, Booth M, Okely A. The reliability of the Adolescent Sedentary Activity Questionnaire (ASAQ). Prev. Med. 2007;45(1):7174. DoI: https://doi.org/10.1016/j.ypmed.2007.03.014

25. Guimarães RdF, Silva MPd, Legnani E, et al. Reproducibility of Adolescent Sedentary Activity Questionnaire (ASAQ) in Brazilian adolescents. Rev Bras de Cineantropom Desempenho Hum. 2013;15(3):276-85. DOI: https://doi.org/10.5007/1980$0037.2013 \mathrm{v} 15 \mathrm{n} 3 \mathrm{p} 276$

26. Zhou J, Zhou Q, Wang DP, et al. [Associations of sedentary behavior and physical activity with dyslipidemia]. Beijing Da Xue Xue Bao Yi Xue Ban [internet]. 2017 [citado 2020 dic. 9]; 49(3):418 23. Disponible en: https://pubmed.ncbi.nlm.nih.gov/28628141/

27. Arango-Vélez EF, Echavarría-Rodríguez AM, et al. Validación de dos cuestionarios para evaluar el nivel de actividad física y el tiempo sedentario en una comunidad universitaria de Colombia. Rev Fac Nac Salud Pública. 2020;38(1):e334156. Dor: https://doi. org/10.17533/udea.rfnsp.v38n1e334156.

28. Organización Mundial de la Salud. Actividad física. Ginebra [internet]; 2020 [citado 2020 dic. 9]. Disponible en: http://www.who. int/mediacentre/factsheets/fs385/es/
29. Sawka KJ, McCormack GR, Nettel-Aguirre A, et al. Associations between aspects of friendship networks, physical activity, and sedentary behaviour among adolescents. J Obesity. 2014; Article ID 632689. DOI: https://doi.org/10.1155/2014/632689

30. Borgatti SP, Everett MG, Freeman LC. Ucinet for Windows: Software for Social Network Analysis. Harvard, mA: Analytic Technologies [internet]; 2002 [citado 2019 dic. 10]. Disponible en: https://sites.google.com/site/ucinetsoftware/home

31. Asociación Médica Mundial (АмM). Declaración de Helsinki de la AMM - Principios éticos para las investigaciones médicas en seres humanos [internet]; 2013 [citado 2017 feb. 4]. Disponible en: https:/www.wma.net/es/policies-post/declaracion-de-helsinkide-la-amm-principios-eticos-para-las-investigaciones-medicasen-seres-humanos/

32. Colombia, Ministerio de Salud. Resolución 8430 por la cual se establecen las normas científicas, técnicas y administrativas para la investigación en salud (1993 oct. 4).

33. Guerrero Pepinosa NY, Muñoz Ortiz RF, Muñoz Martínez AP, et al. Nivel de sedentarismo en los estudiantes de Fisioterapia de la Fundación Universitaria María Cano, Popayán. Hacia Promoc Salud. 2015;20(2):77-89. DoI: https://doi.org/10.17151/hpsal.2015.20.2.5

34 Marks J, de la Haye K, Barnett LM, Allender S. Friendship network characteristics are associated with physical activity and sedentary behavior in early adolescence. PLoS One. 2015;10(12):e0145344. DOI: https://doi.org/10.1371/journal.pone.0145344

35. Martínez L E, Saldarriaga JF, Sepúlveda FÉ. Actividad física en Medellín: desafío para la promoción de la salud. Rev Fac Nac Salud Pública [internet]. 2008 [citado 2019 dic. 20]; 26(2):117-23. Disponible en: https://revistas.udea.edu.co/index.php/fnsp/article/ view/840/733

36. Rauner A, Mess F, Woll A. The relationship between physical activity, physical fitness and overweight in adolescents: A systematic review of studies published in or after 2000. BMC Pediatr. 2013;13:19. DoI: https://doi.org/10.1186/1471-2431-13-19

37. Casajús JA, Leiva MT, Ferrando JA, et al. Relación entre la condición física cardiovascular y la distribución de grasa en niños y adolescentes. Apunt. Medicina de l'Esport [internet]. 2006 [citado 2020 dic. 12]; 41(149):7-14. Disponible en: https://www.apunts. org/en-pdf-X0213371706889716

38. Contreras Fernández JJ, Espinoza Aravena RM, Dighero Eberhard B, et al. Actitud sedentaria y factores asociados en estudiantes de Medicina. Rev And Med Deporte [internet]; 2009 [citado 2019 dic.13]; 2(4):133-40. Disponible en: https://www.elsevier. es/es-revista-revista-andaluza-medicina-del-deporte-284-pdfX1888754609462007 